\title{
Prevalence and molecular identification of Borrelia spirochetes in Ixodes granulatus ticks collected from Rattus losea on Kinmen Island of Taiwan
}

\author{
Li-Lian Chao', Li-Ling Liu ${ }^{1}$ and Chien-Ming Shih ${ }^{1,2^{*}}$
}

\begin{abstract}
Background: Ixodes granulatus is widely distributed in various countries of Southeast Asia and Taiwan. Although this tick species is presumed to be the vector for the enzoonotic transmission of Borrelia spirochetes in the Taiwan area, the prevalence of infection and genetic diversity of Borrelia spirochetes harbored by this tick species need to be further determined.

Methods: DNA extraction was performed from individual tick specimens collected from Rattus losea on Kinmen Island of Taiwan. Borrelia infection in I. granulatus ticks was detected by performing a specific PCR assay based on the 5S-23S intergenic spacer amplicon gene of B. burgdorferi sensu lato. The genetic identities of detected spirochetes were identified by gene sequencing and phylogenetic analysis.

Results: Borrelia infection was detected in nymph, male, and female stages of Ixodes granulatus ticks with an infection rate of $42.9 \%, 36 \%$, and $52.7 \%$, respectively. Genospecies identification reveals that $B$. valaisiana is the main genotype (70.7\%) as compared to the genotype of B. burgdorferi sensu stricto (15.4\%). Phylogenetic analysis revealed that these detected spirochetes were genetically affiliated to the genospecies $B$. valaisiana and $B$. burgdorferi sensu stricto, with a high sequence homology within the genospecies of B. valaisiana (95.8 to 100\%) and B. burgdorferi sensu stricto (97.2 to $100 \%)$, respectively.

Conclusions: This study highlights the significance of high prevalence and genetic diversity of Borrelia spirochetes in I. granulatus ticks collected from Rattus losea on Kinmen Island of Taiwan. Intraspecific analysis also revealed that B. valaisiana species detected in Kinmen Island can be easily distinguished from the European group of B. valaisiana and other genospecies of Borrelia spirochetes. This may imply an enzoonotic cycle between I. granulatus ticks and rodent hosts that maintains Borrelia spirochetes in Kinmen Island as well as Southeast Asia.
\end{abstract}

Keywords: Borrelia, Ixodes granulatus, Tick, Genetic diversity, Taiwan

\section{Background}

Lyme disease spirochetes, Borrelia burgdorferi sensu lato, was first identified within the gut of vector ticks [1] and the spirochete species can be classified into at least thirteen genospecies based on their genetic differences [2-5]. The tick species of Ixodes ricinus complex serve as

\footnotetext{
* Correspondence: cmshih@ndmctsgh.edu.tw

${ }^{1}$ Graduate Institute of Pathology and Parasitology, Department of

Parasitology and Tropical Medicine, National Defense Medical Center, Taipei, Taiwan, Republic of China

${ }^{2}$ Research Center for Biotechnology and Medicine Policy, Taipei, Taiwan, Republic of China
}

(c) 2012 Chao et al.; licensee BioMed Central Ltd. This is an Open Access article distributed under the terms of the Creative Commons Attribution License (http://creativecommons.org/licenses/by/2.0), which permits unrestricted use, distribution, and reproduction in any medium, provided the original work is properly cited. the main vectors for transmission and perpetuation of $B$. burgdorferi spirochetes through a natural cycle between vector ticks and rodent hosts in North America and Europe [6,7]. Although I. persulcatus and I. ovatus have been recognized as the principle vector for the transmission of B. burgdorferi spirochetes in Northeast Asia, including the northeastern regions of China, Korea, and Japan [8-11], the hard ticks of I. granulatus, Haemaphysalis longicornis, and $H$. bispinosa were suggested as the principle vectors for the transmission of B. burgdorferi 
spirochetes in the southwestern regions (adjacent to Taiwan) of China [12,13].

The abundance and widespread distribution of I. granulatus has been recorded for the first time from various countries in Southeast Asia and Taiwan [14]. The medical importance with the recent emergence of human babesiosis [15] and Lyme borreliosis [16] in Taiwan raises the focus of research attention on I. granulatus ticks. Indeed, Lyme disease spirochetes (B. burgdorferi sensu lato) have been isolated from six species of rodent hosts in Taiwan [17] and all these Taiwan isolates were genetically classified into the genospecies of B. burgdorferi sensu stricto $[18,19]$. In addition, Borrelia infection has also been detected in I. granulatus ticks collected from the Kinmen Island of Taiwan [20]. Although the hard tick of I. granulatus was presumed to be the tick vector for the enzoonotic transmission of Borrelia spirochetes in the Taiwan area [21], the prevalence of infection and genetic diversity of Borrelia spirochetes harbored by this tick species in Kinmen Island needs to be further defined.

The 5S (rrf)-23S ( $r r l)$ intergenic spacer amplicon gene is unique and highly conserved in $B$. burgdorferi sensu lato $[22,23]$. The diversity of this gene is useful for distinguishing the genetic heterogeneity among different Borrelia isolates [24-26]. Indeed, genetic identity of Borrelia spirochetes was clarified by analyzing the sequence homology of 5S (rrf)-23S ( $r r l)$ intergenic spacer amplicon genes of $B$. burgdorferi sensu lato isolated from various biological sources $[2,13,27,28]$. In addition, different genospecies of $B$. burgdorferi sensu lato are distributed unevently throughout the world and are associated with distinct ecologic features [2]. It may be that the Borrelia spirochetes that exist in I. granulatus ticks of Kinmen Island are genetically affiliated to the genospecies discovered in Asia, which are distinct from the Borrelia spirochetes within common vector ticks (I. ricinus complex) discovered in Europe and the United States. Thus, the objectives of the present study intend to determine the prevalence of Borrelia spirochetes within I. granulatus ticks by polymerase chain reaction (PCR) assay targeting the 5S (rrf)-23S ( $r r l)$ intergenic spacer amplicon gene of B. burgdorferi sensu lato and to clarify the genetic identity of detected spirochetes by analyzing phylogenetic relationships with other Borrelia species that have been documented in GenBank.

\section{Methods}

\section{Collection and identification of tick specimens}

All specimens of adult and nymphal ticks were removed from rodents captured at various field sites of four townships in Kinmen Island (Figure 1). All field-collected ticks were subsequently stored in separate mesh-covered and plaster-bottomed vials. Adult and nymphal ticks of I. granulatus collected from Kinmen Island of Taiwan were identified to species level on the basis of their morphological characteristics, as described previously [29]. In addition, ultrastructural observations by scanning electron microscope (SEM) were also used to identify the morphological features of I. granulatus ticks, as described previously [29].

\section{DNA extraction from tick specimens}

Total genomic DNA was extracted from individual tick specimens used in this study. Briefly, tick specimens were cleaned by sonication for 3-5 min in $75 \%$ ethanol and then washed twice in sterile distilled water. Afterwards, each individual tick specimen was dissected into pieces, placed in a microcentrifuge tube filled with $180-\mu \mathrm{l}$ lysing buffer solution supplied in the DNeasy Blood \& Tissue Kit (catalogue no. 69506, Qiagen, Hilden, Germany) and then homogenized with a sterile tissue grinder (catalogue no. 358103, Wheaton Scientific Products, Millville, NJ, USA). The homogenate was centrifuged at room temperature and the supernatant fluid was further processed using a Dneasy Blood \& Tissue Kit, as per manufacturer's instructions. After filtration, the filtrate was collected and the DNA concentration was determined spectrophotometrically with a DNA calculator (GeneQuant II; Pharmacia Biotech, Uppsala, Sweden).

\section{DNA amplification by polymerase chain reaction (PCR)}

DNA samples extracted from the tick specimens were used as a template for PCR amplification. A nested PCR was performed with primers designed to amplify the variable spacer region between two conserved duplicate structures. A specific primer set corresponding to the 3' end of the 5S rRNA (rrf) (5'-CGACCTTCTTCGCCT TAAAGC-3') and the 5' end of the 23S rRNA ( $r r l)$ (5'TAAGCTGACTAATACTAATTACCC-3') was designed and applied for the primary amplification, as described previously [23]. In the nested PCR, a primer set of primer 1 (5'-CTGCGAGTTCGCGGGAGA-3') and primer 2 (5'TCCTAGGCATTCACCATA-3') was used and expected to yield a 226-266 bp fragment depending on the Borrelia strain, as described previously [30]. All PCR reagents and Taq polymerase were obtained and used as recommended by the supplier (Takara Shuzo Co., Ltd., Japan). Briefly, a total of $0.2-\mu \mathrm{mol}$ of the appropriate primer set and various amounts of template DNA (0.1$0.3 \mu \mathrm{g})$ were used in each $50-\mu \mathrm{l}$ reaction mixture. The PCR amplification was performed with a Perkin-Elmer Cetus thermocycler (GeneAmp system 9700; Applied Biosystems, Taipei, Taiwan), and the primary amplification included 2 min denaturation at $96^{\circ} \mathrm{C}$ followed by 30 cycles of the following conditions: denaturation at $94^{\circ} \mathrm{C}$ for $30 \mathrm{~s}$, annealing at $55^{\circ} \mathrm{C}$ for $30 \mathrm{~s}$, and extension at $72^{\circ} \mathrm{C}$ for $40 \mathrm{~s}$. Nested amplification was performed under the same conditions, except for annealing at $59^{\circ} \mathrm{C}$ for $30 \mathrm{~s}$. 


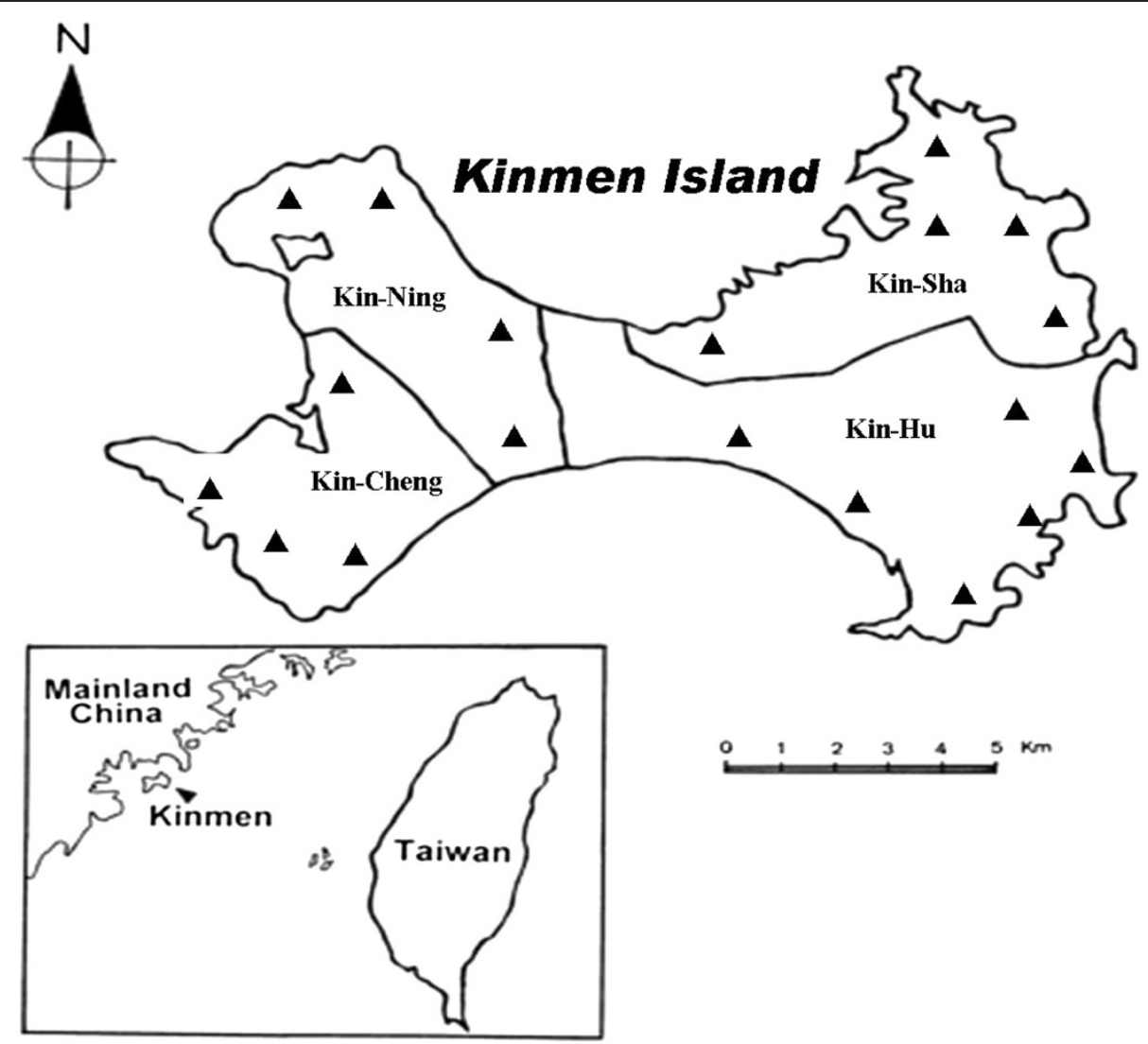

Figure 1 Map of Taiwan and Kinmen Island, showing the geographic location of Kinmen Island and the collection sites ( $\mathbf{\Lambda})$ for I. granulatus ticks removed from captured rodents.

Thereafter, amplified DNA products were electrophoresed on $2 \%$ agarose gels in Tris-Borate-EDTA (TBE) buffer and visualized under ultraviolet (UV) light after staining with ethidium bromide. A DNA ladder (1-kb plus, catalogue no. 10787-018, Gibco BRL, Taipei, Taiwan) was used as the standard marker for comparison. A negative control of distilled water was included in parallel with each amplification.

\section{Sequence alignments and phylogenetic analysis}

After purification with a QIAquick PCR purification kit (catalogue no. 28104, Qiagen, Hilden, Germany), sequencing reactions were performed with 25 cycles under the same conditions and same primer set (primer 1 and primer 2) of nested amplification by using the Big Dye Terminator Cycle Sequencing Kit (V3.1) under an ABI Prism 377-96 DNA sequencer (Applied Biosystems, Foster City, CA, USA). The resulting sequences (241-245 bp) were initially edited by BioEdit software (V5.3) and aligned with the CLUSTAL W software [31]. Afterwards, the aligned sequences (205-209 bp) were further analyzed by comparing with other Borrelia sequences based on the type-strain of different genospecies and different geographic origin of Borrelia spirochetes that were available in GenBank. Phylogenetic analysis was performed by neighbour-joining (NJ) compared with maximum parsimony (MP) methods to estimate the phylogeny of the entire alignment using MEGA 4.0 software [32]. A similarity matrix was constructed using DNASTAR program (Lasergene, version 8.0). All phylogenetic trees were constructed and performed with 1000 bootstrap replications to evaluate the reliability of the constructions, as described previously [33].

\section{Nucleotide sequence accession numbers}

The nucleotide sequences of PCR-amplified 5S ( $r r f)$-23S $(\mathrm{rrl})$ intergenic spacer amplicon genes of Borrelia spirochetes determined in this study have been registered and assigned the following GenBank accession numbers: strains KC-44 (JF970243), KH-05 (JF970244), KH-13 (JF970245), KH-74 (JF970246), KN-11 (JF970247), KS-61 (JF970248), KS-62(JF970249), KH-58(JF970250), KC-14(JF970251), KC-49 (JF970252), KH-70 (JF970253), KH-71 (JF970254), KS-18 (JF970255), and KS-39 (JF970256). For phylogenetic analysis, nucleotide sequences of $5 \mathrm{~S}(r r f)$-23S ( $r r l)$ intergenic spacer amplicon genes from another 19 strains of Borrelia species were included for comparison (Table 1). 
Table 1 Genospecies and strains of Borrelia spirochetes used for analysis in this study

\begin{tabular}{|c|c|c|c|}
\hline \multirow[t]{2}{*}{ Genospecies and strain } & \multicolumn{2}{|c|}{ Origin of Borrelia strain } & \multirow{2}{*}{$\begin{array}{l}\text { 5S (rrf)-23S (rrl) gen } \\
\text { accession number }\end{array}$} \\
\hline & Biological & Geographic & \\
\hline \multicolumn{4}{|l|}{ Taiwan strains } \\
\hline$K C-44$ & Ixodes granulatus & Kin-Cheng, Kinmen & JF970243 \\
\hline $\mathrm{KH}-05$ & 1. granulatus & Kin-Hu, Kinmen & JF970244 \\
\hline $\mathrm{KH}-13$ & 1. granulatus & Kin-Hu, Kinmen & JF970245 \\
\hline $\mathrm{KH}-74$ & 1. granulatus & Kin-Hu, Kinmen & JF970246 \\
\hline $\mathrm{KN}-11$ & 1. granulatus & Kin-Ning, Kinmen & JF970247 \\
\hline KS-61 & 1. granulatus & Kin-Sha, Kinmen & JF970248 \\
\hline KS-62 & 1. granulatus & Kin-Sha, Kinmen & JF970249 \\
\hline $\mathrm{KH}-58$ & 1. granulatus & Kin-Hu, Kinmen & JF970250 \\
\hline KC-14 & 1. granulatus & Kin-Cheng, Kinmen & JF970251 \\
\hline KC-49 & 1. granulatus & Kin-Cheng, Kinmen & JF970252 \\
\hline $\mathrm{KH}-70$ & 1. granulatus & Kin-Hu, Kinmen & JF970253 \\
\hline $\mathrm{KH}-71$ & 1. granulatus & Kin-Hu, Kinmen & JF970254 \\
\hline KS-18 & 1. granulatus & Kin-Sha, Kinmen & JF970255 \\
\hline \multicolumn{4}{|l|}{ B. valaisiana } \\
\hline KS-39 & 1. granulatus & Kin-Sha, Kinmen & JF970256 \\
\hline VS116 & 1. ricinus & Switzerland & L30134 \\
\hline UK & 1. ricinus & England & L30133 \\
\hline QLZSP1 & I. granulatus & China & EU247839 \\
\hline QTDM2 & 1. granulatus & China & EU429347 \\
\hline CKA2a & Apodemus agrarius & China & AB022124 \\
\hline OG1/01 & 1. granulatus & Japan & AB091441 \\
\hline OG45/01 & 1. granulatus & Japan & AB091455 \\
\hline HN6 & 1. granulatus & Korea & AF058705 \\
\hline \multicolumn{4}{|l|}{ B. burgdorferi sensu stricto } \\
\hline B31 & I. scapularis & USA & L30127 \\
\hline JD1 & I. scapularis & USA & AY032911 \\
\hline TWKM5 & Rattus norvegicus & Taiwan & AY032908 \\
\hline \multicolumn{4}{|l|}{ B. garinii } \\
\hline 20047 & 1. ricinus & France & L30119 \\
\hline NP81 & I. persulcatus & Japan & D84406 \\
\hline \multicolumn{4}{|l|}{ B. afzelii } \\
\hline VS461 & 1. ricinus & Switzerland & L30135 \\
\hline PGau & Human skin & Germany & DQ111066 \\
\hline \multicolumn{4}{|l|}{ B. bissettii } \\
\hline DN127 & 1. pacificus & USA & L30126 \\
\hline CA376 & Neotoma fuscipes & USA & AY177634 \\
\hline \multicolumn{4}{|l|}{ B. sinica } \\
\hline CMN1a & Niviventer sp. & China & AB022129 \\
\hline CMN3 & Niviventer sp. & China & AB022131 \\
\hline
\end{tabular}

${ }^{\mathrm{a} G e n B a n k}$ accession numbers (JF970243 JF970256) were submitted by this study. 


\section{Results}

PCR detection of Borrelia infection in I. granulatus ticks

To verify the existence of Borrelia spirochetes in I. granulatus ticks removed from rodents of Kinmen Island. A total of 292 field rodents (Rattus losea) were captured and examined for I. granulatus ticks from four townships of Kinmen Island. An overall infestation was observed on $62 \%(181 / 292)$ captured rodents with an average density of 1.44 ticks per infested rodent. A total of 261 ticks (131 female, 25 male, and 105 nymph) were examined and tested for the evidence of spirochete infection by PCR using specific primers targeting the $5 \mathrm{~S}(r r f)$-23S ( $r r l)$ intergenic spacer amplicon genes of $B$. burgdorferi sensu lato. Results indicate that Borrelia infections were detected in $52.7 \%(69 / 131)$ of females, $36 \%(9 / 25)$ of males, and $42.9 \%(45 / 105)$ of nymphs of $I$. granulatus ticks (Table 2). The highest infection was detected in 52\% (65/ 125) of I. granulatus ticks collected from Kin-Hu township. In contrast, the lowest infection was detected in $12.5 \%(1 / 8)$ of $I$. granulatus ticks collected from KinNing township. The overall infection was detected in $47.1 \%(123 / 261)$ of I. granulatus ticks collected from Kinmen Island.

\section{Genetic identification of detected spirochetes}

To clarify the genetic identity of Borrelia spirochetes detected in I. granulatus ticks collected from Kinmen Island, sequences of PCR-amplified 5S ( $r r f)$-23S ( $r r l)$ intergenic spacer fragments of 123 strains of Borrelia spirochetes were aligned and compared with the downloaded sequences of known genospecies of Borrelia spirochetes. Results indicate that B. valaisiana was detected as the main genotype in $70.7 \%$ (87/123) of I. granulatus ticks and the genotype of $B$. burgdorferi sensu stricto was also detected in 15.4\% (19/123) of I. granulatus ticks (Table 3). However, there still are 13.8\% (17/123) of untyped strains.

\section{Sequence analysis of detected spirochetes}

Sequence similarity of PCR-amplified 5S ( $r r f)$-23S ( $r r l)$ intergenic spacer fragments of 14 selected strains of
Borrelia spirochetes from Kinmen Island were aligned and compared with the downloaded sequences of another 19 strains of Borrelia spirochetes (8 B. valaisiana, 3 B. burgdorferi sensu stricto, 2 B. garinii, 2 B. afzelii, 2 $B$. bissettii, and 2 B. sinica) from GenBank. The nucleotide sequences between the 14 Borrelia spirochetes of Kinmen Island are highly homogeneous with a high sequence homology within the genospecies of $B$. burgdorferi sensu stricto (97.2 to 100\%) and B. valaisiana (95.8 to 100\%), respectively (Table 4). However, intraspecific analysis based on the sequence similarity reveals that all these B. valaisiana spirochetes (GenBank accession numbers: JF970243, JF970246, JF970248-9, and JF970251-6) of Kinmen Island are closely related with the Asian group (China, Japan, and Korea) of $B$. valaisiana (GenBank accession numbers: EU247839, EU429347, AB022124, AB091441, AB091455, and AF058705) and can be distinguished from the European group (Switzerland and England) of B. valaisiana (GenBank accession numbers: L30133 and L30134), as well as other genospecies of $B$. burgdorferi sensu lato (Table 4).

\section{Phylogenetic analysis of detected spirochetes}

Phylogenetic relationships based on the alignment of $5 \mathrm{~S}$ $(r r f)$-23S $(r r l)$ intergenic spacer sequences were performed to analyze the genetic divergence among 33 Borrelia spirochetes investigated in this study. Bootstrap analysis was used to analyze the repeatability of the clustering of specimens represented in phylogenetic trees. Phylogenetic trees constructed by both NJ (Figure 2) and MP (data not shown) analyses showed congruent basal topologies with nine major branches of distinguished clades. All Borrelia spirochetes detected in I. granulatus ticks from Kinmen Island represent two major groups of B. valaisiana spirochetes (groups A-B) which constituted a separate clade that can be easily distinguished from the European group of B. valaisiana spirochetes, and one major group of Borrelia spirochetes which was highly affiliated with the main genospecies of $B$. burgdorferi

Table 2 Detection of Borrelia infection in various stages of $I$. granulatus ticks collected from four townships of Kinmen Island by PCR assay targeting the 5S (rrf)-23S (rrl) intergenic spacer gene of B. burgdorferi sensu lato

\begin{tabular}{|c|c|c|c|c|}
\hline \multirow[t]{3}{*}{ Township } & \multicolumn{3}{|c|}{ Stage of tick ${ }^{a}$} & \multirow{3}{*}{$\begin{array}{c}\text { Total No. infected/No. } \\
\text { tested (\%) }\end{array}$} \\
\hline & Male & Female & Nymph & \\
\hline & No. infected/No. tested (\%) & No. infected/No. tested (\%) & No. infected/No. tested (\%) & \\
\hline Kin-Hu & 6/14 (42.9) & $40 / 65(61.5)$ & 19/46 (41.3) & $65 / 125(52.0)$ \\
\hline Kin-Sha & 3/9 (33.3) & 25/57 (43.9) & 19/36 (52.8) & 47/102 (46.1) \\
\hline Kin-Ning & $0 / 0(0.0)$ & $1 / 3(33.3)$ & $0 / 5(0.0)$ & $1 / 8(12.5)$ \\
\hline Kin-Cheng & $0 / 2(0.0)$ & $3 / 6(50.0)$ & $7 / 18$ (38.9) & 10/26 (38.5) \\
\hline Total & $9 / 25(36.0)$ & $69 / 131(52.7)$ & $45 / 105$ (42.9) & $123 / 261(47.1)$ \\
\hline
\end{tabular}

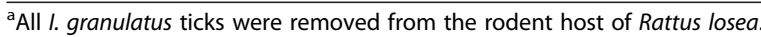


Table 3 Genospecies identification of Borrelia spirochetes detected in I. granulatus ticks collected from four townships of Kinmen Island, Taiwan

\begin{tabular}{lcccc}
\hline Township & Positive No. by PCR & \multicolumn{3}{c}{ Genospecies determined by 5S (rrf)-23S (rrl) gene sequences } \\
\cline { 2 - 5 } & & B. burgdorferi sensu stricto & B. valaisiana & Untyping \\
\hline Kin-Hu & 65 & 12 & 46 & 7 \\
Kin-Sha & 47 & 4 & 0 & 8 \\
Kin-Ning & 1 & 1 & 6 & 0 \\
Kin-Cheng & 10 & 2 & $87(70.7)$ & $17(13.8)$ \\
\hline Total (\%) & 123 & $19(15.4)$ &
\end{tabular}

sensu stricto (strains B31, JD1, and TWKM5) (Figure 2). The phylogenetic analysis of NJ tree strongly supports the separation of different lineages between the Borrelia spirochetes from Kinmen Island and Europe with a bootstrap value of 84 . These results reveal a lower genetic divergence within the same genospecies of Borrelia spirochetes from Kinmen Island of Taiwan, but a higher genetic variation among different genospecies or variant geographic origins of Borrelia spirochetes.

\section{Discussion}

This study describes the first survey regarding the prevalence and genetic identification of Borrelia spirochetes detected in various stages of I. granulatus ticks collected from the offshore Kinmen Island of Taiwan. In our previous investigations, $B$. burgdorferi spirochetes had been isolated from six species of rodent hosts captured at various locations in Taiwan and I. granulatus ticks were observed on four species of highly infested rodent hosts [17]. Although the existence of zoonotic transmission of Borrelia spirochetes was suggested, the identification of Borrelia spirochetes within possible vector tick is required to verify the natural infection in Kinmen Island. Indeed, results from the present study confirm the high prevalence of $B$. burgdorferi sensu stricto and B. valaisiana spirochetes detected in various stages of I. granulatus ticks (Tables 2 and 3), and reveal that the rodent species of $R$. losea serves as the major infested host for maintaining the natural transmission of Borrelia spirochetes in Kinmen Island of Taiwan. Further investigations focusing on the seasonal abundance and prevalence of spirochetal infections among reservoir hosts would help to elucidate the enzoonotic transmission of Borrelia spirochetes in Kinmen Island of Taiwan.

Table 4 Sequence similarity between $5 S$ ( $r r f)-23 S(r r l)$ gene sequences from Taiwan strains of Borrelia detected in Ixodes granulatus ticks and strains of other genospecies of Borrelia

\begin{tabular}{|c|c|c|c|c|c|c|c|c|c|c|c|c|c|c|c|c|c|}
\hline Genospecies and strain ${ }^{a}$ & B31 & TWKM5 & KH5 & KN11 & KH13 & UK & OG1 & HN6 & QLZSP1 & KC44 & $\mathrm{KH} 74$ & KS61 & KS62 & 20047 & VS461 & DN127 & CMN1a \\
\hline Bbss B31 & - & 99.1 & 99.1 & 97.2 & 97.7 & 89.3 & 90.7 & 90.7 & 90.7 & 90.7 & 90.2 & 90.7 & 90.2 & 93.0 & 86.0 & 92.1 & 81.8 \\
\hline Bbss TWKM5 & & - & 100 & 98.1 & 98.6 & 90.2 & 90.7 & 90.7 & 90.7 & 90.7 & 90.2 & 90.7 & 90.2 & 93.0 & 86.0 & 92.1 & 81.8 \\
\hline KH5 & & & - & 98.1 & 98.6 & 90.2 & 90.7 & 90.7 & 90.7 & 90.7 & 90.2 & 90.7 & 90.2 & 93.0 & 86.0 & 92.1 & 81.8 \\
\hline KN11 & & & & - & 98.6 & 89.3 & 89.7 & 89.7 & 89.7 & 89.7 & 89.3 & 89.7 & 89.3 & 92.1 & 85.5 & 90.2 & 79.9 \\
\hline $\mathrm{KH} 13$ & & & & & - & 88.8 & 89.3 & 89.3 & 89.3 & 89.3 & 88.8 & 89.3 & 88.8 & 91.6 & 84.6 & 90.7 & 81.3 \\
\hline$B V U K$ & & & & & & - & 95.8 & 95.8 & 95.8 & 95.8 & 96.3 & 95.8 & 95.8 & 93.0 & 87.4 & 88.3 & 80.8 \\
\hline$B \vee O G 1$ & & & & & & & - & 100 & 100 & 99.1 & 99.5 & 99.1 & 99.1 & 93.5 & 88.3 & 87.9 & 80.4 \\
\hline BV HNG & & & & & & & & - & 100 & 99.1 & 99.5 & 99.1 & 99.1 & 93.5 & 88.3 & 87.9 & 80.4 \\
\hline BV QLZSP1 & & & & & & & & & - & 99.1 & 99.5 & 99.1 & 99.1 & 93.5 & 88.3 & 87.9 & 80.4 \\
\hline KC44 & & & & & & & & & & - & 99.5 & 100 & 99.1 & 93.5 & 88.3 & 88.8 & 81.3 \\
\hline KH74 & & & & & & & & & & & - & 99.5 & 99.5 & 93.0 & 87.9 & 88.3 & 80.8 \\
\hline KS61 & & & & & & & & & & & & - & 99.1 & 93.5 & 88.3 & 88.8 & 81.3 \\
\hline KS62 & & & & & & & & & & & & & - & 93.0 & 87.9 & 88.3 & 80.8 \\
\hline Bg 20047 & & & & & & & & & & & & & & - & 90.7 & 90.7 & 82.7 \\
\hline Ba VS461 & & & & & & & & & & & & & & & - & 85.5 & 82.7 \\
\hline Bbis DN127 & & & & & & & & & & & & & & & & - & 79.9 \\
\hline Bs CMN1a & & & & & & & & & & & & & & & & & - \\
\hline
\end{tabular}

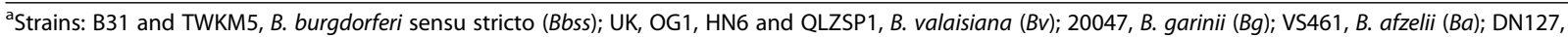
B. bissettii (Bbis); CMN1a, B. sinica (Bs). 


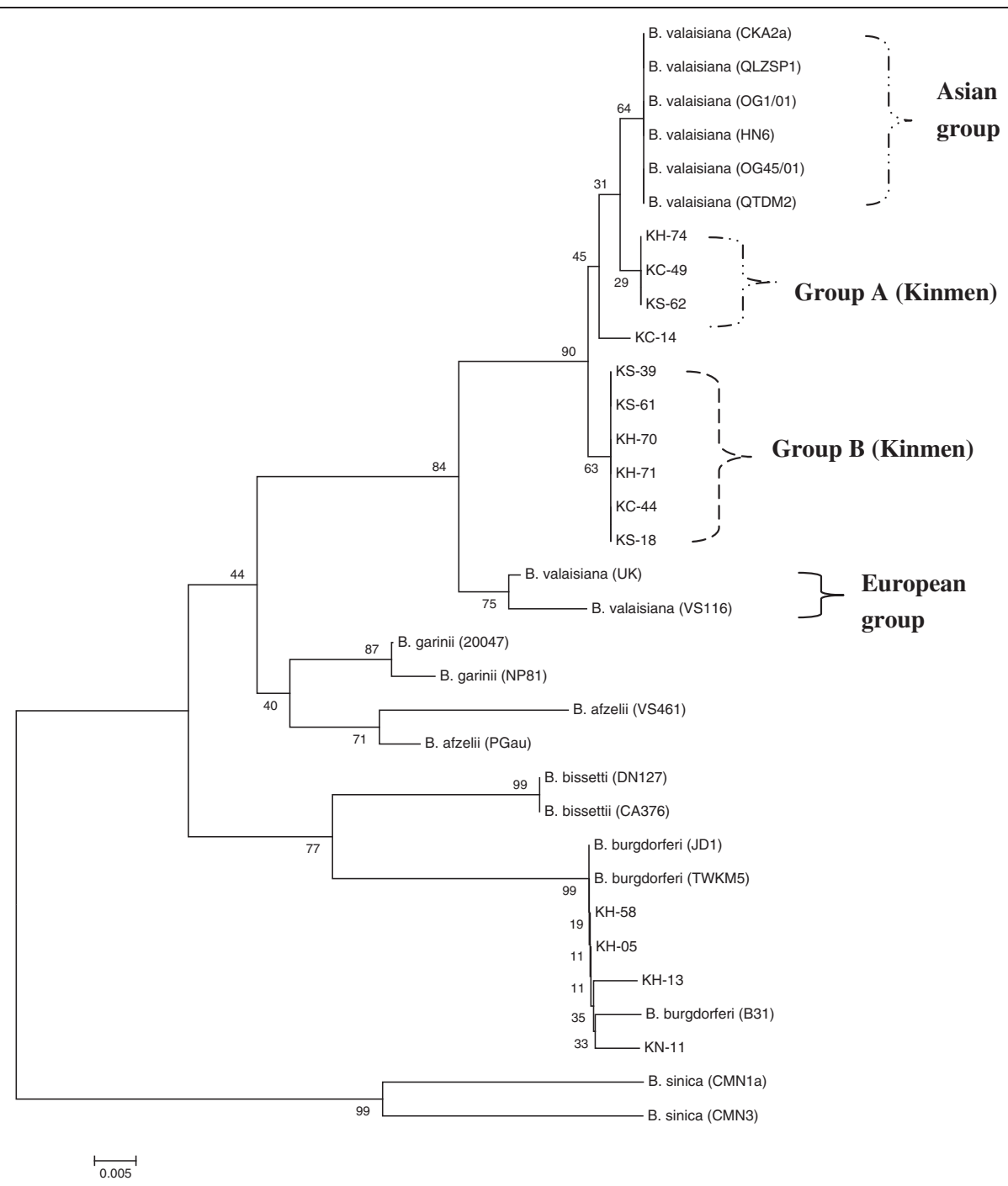

Figure 2 Phylogenetic relationships based on the aligned sequences (205-209 bp) of 5S (rrf)-23S (rrl) rRNA gene were used to compare the genetic variation between 14 Borrelia spirochetes detected in I. granulatus ticks from Kinmen Island of Taiwan and 19 other strains of Borrelia spirochetes. The tree was constructed and analyzed by the neighbour-joining method using 1000 bootstrap replicates. Numbers at the nodes indicate the percentages of reliability of each branch of the tree. Branch lengths are drawn proportional to the estimated sequence divergence.

The transmission cycle for Borrelia spirochetes in Southeast Asia remains elusive. It is assumed that different genospecies of Borrelia spirochetes is relevant to the distinct reservoir hosts and vector ticks [2]. Indeed, $B$. valaisiana has been isolated or detected from I. ricinus ticks and avain reservoirs from at least eight European countries [34-39]. In addition, B. valaisiana-related spirochetes were isolated mainly from rodent hosts and detected in various hard ticks (I. nipponensis, I. columnae, I. granulatus, and Haemaphysalis longicornis) in Northeast Asia and Southwestern China [13,40-42]. However, I. granulatus is widespread in various countries of Southeast Asia and Taiwan [14]. Results from this study also verify the high prevalence of B. valaisiana and B. burgdorferi sensu stricto detected in I. granulatus ticks that are infested on the rodent host of $R$. losea in Kinmen Island. These observations may suggest that an enzoonotic cycle between rodent hosts and I. granulatus ticks exists, therefore perpetuating Borrelia spirochetes in Kinmen Island and Southeast Asia.

The existence of two tandemly duplicated copies of $5 \mathrm{~S}$ $(r r f)$-23S ( $r r l$ intergenic spacer genes in B. burgdorferi spirochetes is unique and has not been found in other eubacteria $[22,23]$. Taking advantage of this unique genomic character, the genetic identity of Borrelia spirochetes can be distinguished by their differential reactivities with 
genospecies-specific PCR primers targeting the 5S ( $r r f)$ 23S $(r r l)$ intergenic spacer amplicon gene. Indeed, genetic heterogeneity can be further classified among Borrelia isolates that were previously identified as the same genospecies of atypical strains of Borrelia spirochetes $[25,26]$. Results from the present study also verify that the genetic identities of Borrelia spirochetes detected within I. granulatus ticks of Kinmen Island are highly homogeneous within the genospecies of $B$. burgdorferi sensu stricto and B. valaisiana, and were clearly distinguished from other genospecies of Borrelia spirochetes (Tables 2, 3 , 4). Further application of these genospecies-specific PCR tools to analyze the 5S ( $r r f)$-23S ( $r r l)$ genes of Borrelia spirochetes detected in various tick species would help to clarify the genetic divergence of Borrelia spirochetes transmitted in the natural cycle of Kinmen Island.

Phylogenetic relationships among Borrelia spirochetes can be constructed and determined by analyzing their sequence homogeneity of a specific target gene. Indeed, the sequence analysis of $5 \mathrm{~S}(r r f)$-23S $(r r l)$ intergenic spacer amplicon gene among various Borrelia spirochetes had been proved useful to evaluate the taxonomic relatedness of Borrelia spirochetes derived from various biological and geographical sources [24,28,43,44]. Although PCR amplification of the intergenic spacer region located between the $r r f$ and $r r l$ genes of $B$. burgdorferi sensu lato had been reported to generate a DNA fragment of approximately 226-266 bp long [30], the variation of nucleotide sequence depends on the strain or group diversity of Borrelia spirochetes and may actually represent the genetic distance of phylogenetic divergence between or within the genospecies of Borrelia spirochetes $[2,30,44]$. In this study, phylogenetic analysis based on the sequences of $5 \mathrm{~S}(r r f)-23 \mathrm{~S}(r r l)$ intergenic spacer amplicon gene of Borrelia spirochetes from Kinmen Island demonstrated a high sequence homogeneity among Borrelia spirochetes within the genospecies of B. burgdorferi sensu stricto (Table 4; Figure 2). However, a high genetic heterogeneity within the genospecies of $B$. valaisiana spirochetes was also observed between the Asian group and European group of Borrelia strains (Figure 2). Although a low intraspecific variation was observed among the same Asian groups of B. valaisiana, all strains of B. valaisiana from Kinmen Island represented as two separate clades (groups A-B) that can be separated from the Asian group and clearly distinguished from the European group of B. valaisiana (Figure 2). The phylogenetic trees constructed by either NJ or MP analysis strongly support the discrimination recognizing the separation of different lineages of $B$. valaisiana detected from the Asian group, European group, and Kinmen Island of Taiwan. Accordingly, these observations reveal that all these B. valaisiana spirochetes detected in I. granulatus ticks from Kinmen Island represent two major groups forming a unique clade distinct from the European group of B. valaisiana.

\section{Conclusions}

This study provides the first survey regarding the prevalence and genetic diversity of Borrelia spirochetes within I. granulatus ticks collected from Kinmen Island of Taiwan. Further application of this molecular tool to investigate the genetic variability among Borrelia spirochetes detected in different vector ticks and reservoir hosts may facilitate our understanding of the significance of genetic diversity in relation to the epidemiological features of Borrelia spirochetes in Southeast Asia.

\section{Competing interests}

The authors declare that they have no competing interests.

\section{Authors' contributions}

CMS designed the study, and contributed with tick collection, data analysis, interpretation, and manuscript writing. LLC contributed with tick collection, tick identification, sequencing, and phylogenetic analysis. LLL performed DNA extraction, PCR amplification, and gel electrophoresis. All authors read and approved the final version of the manuscript.

\section{Acknowledgements}

This work was supported in part by grants from the National Science Council (NSC99-2314-B-760-001-MY2) and Department of Defense (I-13), Taipei, Taiwan, Republic of China.

Received: 21 June 2012 Accepted: 6 August 2012

Published: 10 August 2012

\section{References}

1. Burgdorfer W, Barbour AG, Hayes HF, Benach $J$, Grunwaldt E, Davis JP: Lyme disease: a tick-borne spirochetosis? Science 1982, 216:1317-1319.

2. Wang G, van Dam AP, Schwartz I, Dankert J: Molecular typing of Borrelia burgdorferi sensu lato: taxonomic, epidemiological, and clinical implications. Clin Microbiol Rev 1999, 12:633-653.

3. Masuzawa T, Takada N, Kudeken M, Fukui T, Yano Y, Ishiguro F, Kawamura Y, Imai Y: Ezaki T. Borrelia sinica sp. Nov., a Lyme disease-related Borrelia species isolated in China. Int J Syst Evol Microbiol 2001, 51:1817-1824.

4. Richter D, Postic D, Sertour N, Livey I: Matuschka FR. Baranton G: Delineation of Borrelia burgdorferi sensu lato species by multilocus sequence analysis and confirmation of the delineation of Borrelia spielmanii sp. nov. Int J Syst Evol Microbiol 2006, 56:873-881

5. Postic D, Garnier M, Baranton G: Multilocus sequence analysis of atypical Borrelia burgdorferi sensu lato isolates: description of Borrelia californiensis sp. nov., and genomospecies 1 and 2. Int J Med Microbiol 2007, 297:263-271.

6. Spielman A: Lyme disease and human babesiosis: evidence incriminating vector and reservoir hosts. In The Biology of Parasitism. Edited by Englund PT, Sher AR. New York, NY: Liss; 1988:147-165.

7. Matuschka FR, Richter D, Fischer P, Spielman A: Subadult Ixodes ricinus (Acari: Ixodidae) on rodents in Berlin, West Germany. J Med Entomol 1990, 27:385-390.

8. Kawabata M, Baba S, Iguchi K, Yamaguchi N, Russell H: Lyme disease in Japan and its possible incriminated tick vector. Ixodes persulcatus. J Infect Dis 1987, 156:854.

9. Ai CX, Wen YX, Zhang YG, Wang SS, Qui SS, Shi ZX, Li DY, Chen DQ, Liu XD, Zhao JH: Clinical manifestations and epidemiological characteristics of Lyme disease in Hailin county, Heilongjiang Province, China. Ann NY Acad Sci 1988, 539:302-313.

10. Nakao M, Miyamoto K, Uchikawa K, Fujita H: Characterization of Borrelia burgdorferi isolated from Ixodes persulcatus and Ixodes ovatus ticks in Japan. Am J Trop Med Hyg 1992, 47:505-511. 
11. Park KH, Chang WH, Schwan TG: Identification and characterization of Lyme disease spirochetes, Borrelia burgdorferi sensu lato, isolated in Korea. J Clin Microbiol 1993, 31:1831-1837.

12. Zhang ZF, Wan KL, Zhang JS, Chu KF, Dou GL, Li MC, Chan L, Liang CK, Hou TS, Wong HI: Studies on epidemiology and etiology of Lyme disease in China. Chinese J Epidemiol 1997, 18:8-11. in Chinese.

13. Chu CY, Liu W, Jiang BG, Wang DM, Jiang WJ, Zhao QM, Zhang PH, Wang ZX, Tang GP, Yang H, Cao WC: Novel genospecies of Borrelia burgdorferi sensu lato from rodents and ticks in southwestern China. J Clin Microbiol 2008, 46:3130-3133.

14. Wilson N: New distributional records of ticks from Southeast Asia and the Pacific (Metastigmata: Argasidae, Ixodidae). Oriental Insects 1970, 4:37-46.

15. Shih CM, Liu LP, Chung WC, Ong SJ, Wang CC: Human babesiosis in Taiwan: asymptomatic infection with a Babesia microti-like organism in a Taiwanese woman. J Clin Microbiol 1997, 35:450-454.

16. Shih CM, Wang JC, Chao LL, Wu TN: Lyme disease in Taiwan: first human patient with characteristic erythema chronicum migrans skin lesion. J Clin Microbiol 1998, 36:807-808.

17. Shih CM, Chao LL: Lyme disease in Taiwan: primary isolation of Borrelia burgdorferi-like spirochetes from rodents in Taiwan area. Am J Trop Med Hyg 1998, 59:687-692.

18. Shih CM, Chang HM, Chen SL, Chao LL: Genospecies identification and characterization of Lyme disease spirochetes of genospecies Borrelia burgdorferi sensu lato isolated from rodents in Taiwan. J Clin Microbiol 1998, 36:3127-3132

19. Shih CM, Chao LL: An OspA-based genospecies identification of Lyme disease spirochetes (Borrelia burgdorferi sensu lato) isolated in Taiwan. Am J Trop Med Hyg 2002, 66:611-615.

20. Chao LL, Wu WJ, Shih CM: First detection and molecular identification of Borrelia burgdorferi-like spirochetes in Ixodes granulatus ticks collected on Kinmen Island of Taiwan. Am J Trop Med Hyg 2009, 80:389-394.

21. Shih CM, Chao LL, et al: Current status of Lyme disease in Taiwan with description of its vector ticks and reservoir hosts, Proceedings, Symposium on rodent damage and control strategy. The Plant Protection Society of the Republic of China, Taichung, Taiwan: In: Lu, KH; 2004:79-92. in Chinese.

22. Fukunaga M, Sohnaka M: Tandem repeat of the $23 \mathrm{~S}$ and $5 \mathrm{~S}$ ribosomal RNA genes in Borrelia burgdorferi, the etiological agent of Lyme disease. Biochem Biophys Res Commun 1992, 183:952-957.

23. Schwartz JJ, Gazumyan Y, Schwartz I: rRNA gene organization in the Lyme disease spirochete, Borrelia burgdorferi. J Bacteriol 1992, 174:3757-3765.

24. Rijpkema SG, Molkenboer M, Schouls LM, Jongejan F, Schellekens JF: Simutaneous detection and genotyping of three genomic groups of Borrelia burgdorferi sensu lato in Dutch Ixodes ricinus ticks by characterization of the amplified intergenic spacer region between $5 \mathrm{~S}$ and 23 S rRNA genes. J Clin Microbiol 1995, 33:3091-3095.

25. Mathiesen DA, Oliver JH, Kolbert CT, Tullson ED, Johnson BJ, Campbell GL, Mitchell PD, Reed KD, Telford SR III, Anderson JF, Lane RS, Persing DH: Genetic heterogeneity of Borrelia burgdorferi in the United States. J Infect Dis 1997, 175:98-107.

26. Postic D, Ras NM, Lane RS, Hendson M, Baranton G: Expanded diversity among California Borrelia isolates and description of Borrelia bissettii sp. nov. (formally Borrelia group DN127). J Clin Microbiol 1998, 36:3497-3504.

27. Rijpkema SG, Tazelaar DJ, Molkenboer MJ, Noordhoek GT, Plantinga G, Schouls LM, Schellekens JF: Detection of Borrelia afzelii, Borrelia burgdorferi sensu stricto, Borrelia garinii and group VS116 by PCR in skin biopsies of patients with erythema migrans and acrodermatitis chrinica atrophicans. Clin Microbiol Infect 1997, 3:109-116.

28. Chao LL, Shih CM: Molecualr characterization of Lyme disease spirochetes (Borrelia burgdorferi sensu lato) isolated in Taiwan by restriction fragment length polymorphism analysis of 5S-23S intergenic spacer amplicons. Am J Trop Med Hyg 2002, 67:504-510.

29. Chao LL, Wu WJ, Shih CM: Molecular analysis of Ixodes granulatus, a possible vector tick for Borrelia burgdorferi sensu lato in Taiwan. Exp Appl Acarol 2009, 48:329-344.

30. Postic D, Assous MV, Grimont PA, Baranton G: Diversity of Borrelia burgdorferi sensu lato evidenced by restriction fragment length polymorphism of $\operatorname{rrf(5S)-rrl(23S)~intergenic~spacer~amplicons.~Int~J~Syst~}$ Bacteriol 1994, 44:743-752.

31. Thompson JD, Higgins DG, Gibson TJ: CLUSTAL W: Improving the sensitivity of progressive multiple sequence alignment through sequence weighting, position-specific gap penalties and weight matrix choice. Nuc Acids Res 1994, 22:4673-4680.

32. Tamura K, Dudley J, Nei M: Kumar S. MEGA4: molecular evolutionary genetics analysis (MEGA) software version 4.0. Mol Biol Evol 2007, 24:1596-1599.

33. Felsenstein J: Confidence limits on phylogenies: an approach using the bootstrap. Evolution 1985, 52:1119-1134.

34. Rijpkema SG, Golubic D, Molkenboer M: Verbeek-De Kruif N, Schellekens J. F: Identification of four genomic groups of Borrelia burgdorferi sensu lato in Ixodes ricinus ticks collected in a Lyme borreliosis endemic region of northern Croatia. Exp Appl Acarol 1996, 20:23-30.

35. Kirstein F, Rijpkema SG, Molkenboer M, Gray JS: The distribution and prevalence of $B$. Burgdorferi genomospecies in Ixodes ricinus ticks in Ireland. Eur J Epidemiol 1997, 13:67-72.

36. Postic D, Korenberg E, Gorelova N, Kovalevski W, Bellenger E, Baranton G: Borrelia burgdorferi sensu lato in Russia and neighbouring countries: high incidence of mixed isolates. Res Microbiol 1997, 148:691-702.

37. Wang G, van Dam AP, Le Fleche A, Postic D, Peter O, Baranton G, de Boer R, Spanjaard L, Dankert J: Genetic and phenotypic analysis of Borrelia valaisiana sp. Nov. (Borrelia genomic groups VS116 and M19). Int J Syst Bacteriol 1997, 47:926-932

38. Clinco M, Padovan D, Murgia R, Poldini L, Frusteri L, van de Pol I, Verbeek-De KN, Rijpkema SG, Maroli M: Rate of infection of Ixodes ricinus ticks with Borrelia burgdorferi sensu stricto, Borrelia garinii, Borrelia afzeli and group VS116 in an endemic focus of Lyme disease in Italy. Eur J Clin Microbiol Infect Dis 1998, 17:90-94.

39. Kurtenbach K, Peacey M, Rijpkema SG: Hoodless AN, Nuttall PA, Randolph SE: Differential transmission of the genospecies of Borrelia burgdorferi sensu lato by game birds and small rodents in England. Appl Environ Microbiol 1998, 64:1169-1174.

40. Masuzawa T, Fukui T, Miyake M, Oh HB, Cho MK, Chang WH, Imai Y, Yanagihara Y: Determination of members of a Borrelia afzelii-related group isolated from Ixodes nipponensis in Korea as Borrelia valaisiana. Int. J Syst Bacterial 1999, 49:1409-1415.

41. Masuzawa T, Hashimoto N, Kudeken M, Kadosaka T, Nakamura M, Kawabata $\mathrm{H}$, Koizumi N, Imai Y: New genomospecies related to Borrelia valaisiana, isolated from mammals in Okinawa archipelago, Japan. $J$ Med Microbiol 2004, 53:421-426.

42. Hao Q, Hou XX, Geng Z, Wan KL: Distribution of Borrelia burgdorferi sensu lato in China. J Clin Microbiol 2011, 49:647-650.

43. Liveris D, Gazumyan A, Schwartz I: Molecular typing of Borrelia burgdorferi sensu lato by PCR-restriction fragment length polymorphism analysis. J Clin Microbiol 1995, 33:589-595.

44. Li MQ, Masuzawa T, Takada N, Ishiguro F, Fujita H, Iwaki A, Wang HP, Wang JC, Kawabata M, Yanagihara Y: Lyme disease Borrelia species in northeastern China resemble those isolated from Far eastern Russia and Japan. Appl Env Microbiol 1998, 64:2705-2709.

doi:10.1186/1756-3305-5-167

Cite this article as: Chao et al:: Prevalence and molecular identification of Borrelia spirochetes in Ixodes granulatus ticks collected from Rattus losea on Kinmen Island of Taiwan. Parasites \& Vectors 2012 5:167.

\section{Submit your next manuscript to BioMed Central and take full advantage of:}

- Convenient online submission

- Thorough peer review

- No space constraints or color figure charges

- Immediate publication on acceptance

- Inclusion in PubMed, CAS, Scopus and Google Scholar

- Research which is freely available for redistribution 